\section{Uptake of lodine-131-labelled Atabrine by Ehrlich Ascites Tumour and by Sarcoma $S-180$ BALB}

ACKERMAN ${ }^{1,2}$ reported a selective incorporation of iodine-131-labelled atabrine by lung and subcutaneous Novikoff hepatoma and Walker carcinosarcoma-256 tumours. In order to provide more information concerning the usefulness of this labelled compound for the localization and diagnosis of malignant tumours, we investigated its uptake by Ehrlich ascites tumours and sarcoma $S .180$ $B A L B$.

A group of $B A L B$ mice was inoculated with Ehrlich ascites tumour cells. Eight days later they were injected subcutaneously with $10 \mu \mathrm{c}$. of iodine-131-labelled atabrine (specific activity, $0.12 \mathrm{mc} . / \mathrm{mg})^{3}$. Groups consisting of five animals each were killed 1,2 and 5 days after the injection. The intraperitoneal exudate was immediately withdrawn with a syringe and centrifuged at 3,000 r.p.m. for $15 \mathrm{~min}$. Both centrifugates and supernatants were counted for radioactivity with a scintillation counter; in addition, the radioactivity in tho liver, spleen and leg muscles was measured. A control group of healthy animals injected with the same dose of iodine-131-labelled atabrine was killed at the same time intervals; the intraperitoneal cavity was washed with distilled water and the water counted for radioactivity under the same conditions. As in the experimental group, the radioactivity retained by the liver, spleen and muscles of control animals was measured. Table 1 gives values corresponding to the specific activities found (c.p.m./g tissue).

A similar injection test was performed on a group of five $B A L B$ mice with sarcoma $S-180 B A L B$. These tumours were produced by inoculating tumour-cells 20 days beforehand. The animals were killed 5 days after the intraperitoneal injection of the iodine-131-labelled atabrine. The subcutaneous tumours were excised and also the adjacent and abdominal muscles. The radioactivity was counted in each case (Table 1).

Table 1. Values of the SPECIFic Aotivity (c.P.M./G Tissuk)

\begin{tabular}{|c|c|c|c|c|}
\hline Fbrlich ascites tun & 1 day & 2 days & \multicolumn{2}{|c|}{5 days } \\
\hline \multicolumn{5}{|c|}{ Iodine-131-labelled atabrine (injected subcutaneously) } \\
\hline Cells (centrifugate)* & $135 \pm 87 \dagger$ & $104 \pm 19$ & $107 \pm$ & 71 \\
\hline \multicolumn{4}{|l|}{ Exudate } & \\
\hline Spleen & $\begin{array}{l}300 \pm 215 \\
332 \pm 150\end{array}$ & $382 \pm 125$ & $81 \pm$ & 35 \\
\hline Liver & $273 \pm 136$ & $317 \pm 125$ & $263 \pm$ & 98 \\
\hline Muscle & $159 \pm 106$ & $72 \pm 21$ & $12 \pm$ & 7 \\
\hline \multicolumn{5}{|l|}{ Control } \\
\hline Intraperitoneal washing* & $93 \pm 15$ & $38 \pm 21$ & $13 \pm$ & 10 \\
\hline Spleen & $380 \pm 265$ & $425 \pm 180$ & $138 \pm$ & 98 \\
\hline Eiver & $426 \pm 105$ & $466 \pm 137$ & $764 \pm 3$ & 365 \\
\hline Muscles & $112 \pm 39$ & $98 \pm 54$ & $68 \pm$ & 29 \\
\hline \multicolumn{5}{|c|}{$\begin{array}{l}\text { Sarcoma, } S-180 B A L B \\
\text { iodine-131-1abelled atabrine (injected intraperitoneally) }\end{array}$} \\
\hline \multirow{2}{*}{\multicolumn{3}{|c|}{$\begin{array}{l}\text { Sarcoma } \\
\text { Muscles (leg) }\end{array}$}} & & \\
\hline & & & $89 \pm$ & 44 \\
\hline \multicolumn{3}{|l|}{ Muscles (abdomen) } & $97 \pm$ & 39 \\
\hline
\end{tabular}

The experimental results revealed that the activity incorporated by the Ehrlich ascites tumour cells remained almost unchanged throughout the experiment; the control group showed no significant activity. The pattern of uptake and elimination by the liver and spleen was similar in both groups and the observed variations between them could be related to the influence of increased fluid content in the body of tumour-bearing animals. The most significant finding was the retention of iodine-131-labellod atabrine by the tumour cells, whereas in the other tissues of normal and ascitic animals the radioactivity decreases considerably. The liver, however, is the exception to the rulo. This organ has previously been shown to be responsible for the elimination of this labelled compound through the gastrointestinal tract ${ }^{3}$.

The sareoma $S-180 B A L B$ showed slightly greater activity than the surrounding leg muscles.
These findings seem to corroborate the preferential uptake of certain aminoacridine compounds by the tumour tissue observed by Ackerman.

Comisión Nacional de Energía Atómica, L. J. ANGHILERI* Buenos Aires, Argentina.

* Present address: Deutsches Krebsforschungszentrum, Institut für Nuklearmedizin, Heidelberg, Germany.

${ }^{1}$ Ackerman, N. B., Surg. Forum, 14, 139 (1963).

${ }^{2}$ Ackerman, N. B., and Shemesh, A., J. Amer. Med. A880c., 187, 832 (1964).

Anghileri, L. J., J. Nucl. Med., 6, 69 (1965).

\section{Interaction of Poly-2-vinylpyridine-1-oxide with Silicic Acid}

The ability of poly-2-vinylpyridine-1-oxide (P204) to prevent the pathogenic action of quartz particles in the lungs of experimental animals and the cytotoxic effect in cell cultures has been repeatedly demonstrated ${ }^{1}$. Its mode of action is uncertain. Apparently it does not act by coating the particles because it is more effective if the P204 is added to the culture or is injected intraperitoneally into the animal than if the dust is pre-treated with it. Weiss and Harvey ${ }^{2}$ suggested that P204 may be oxidized in the tissues to poly-2-vinyl-6-hydroxypyridine-1-oxide which might interact with silicic acid formed by dissolution of the quartz. They demonstrated that stable cationic silicon complexes are formed between 2-hydroxypyridine1 -oxide and orthosilicic acid. We have found that interaction occurs between poly-2-vinylpyridine-1-oxide itself and silicic acid by hydrogen bond formation.

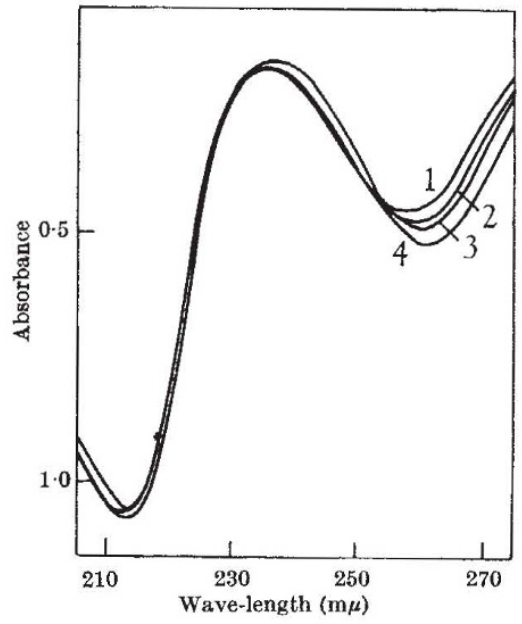

Fig. 1

Poly-2-vinylpyridine-1-oxide in chloroform has an electronic absorption spectrum with a peak at $\lambda_{\max }=$ $272.5 \mathrm{mu}$ (see Fig. 1 and Table 1). In ethanol this peak is shifted to $\lambda_{\max }=268.5 \mathrm{~m} \mu$ and there is an additional peak at $\lambda_{\max }=220 \mathrm{~m} \mu$, the latter being obliterated by chloroform. In water P204 gives peaks at $213 \mathrm{~m} \mu$ and $260.5 \mathrm{~m} \mu$. These values indicate that polyvinylpyridine oxide forms hydrogen bonds with water and with othanol. When the oxide is dissolved in freshly prepared $0.01 \mathrm{M}$ aqueous silicic acid, the peaks are at $213 \mathrm{~m} \mu$ and $259 \mathrm{~m} \mu$. The shift of the longer wavelength peak indicates that the oxide hydrogen bonds more strongly with silicic acid than with

Table 1. ABSORPRION SPECTRA OF POLY-2-VINYLPYRIDINE-1-OXIDE

\begin{tabular}{ccc}
\multicolumn{1}{c}{ Solvent } & $\lambda_{\max }$ & $\boldsymbol{\varepsilon}_{\max }$ \\
& $(\mathrm{m} \mu)$ & \\
Chloroform & $272 \cdot 5$ & $\mathbf{6 , 6 3 6}$ \\
Ethyl alcohol & $268 \cdot 5$ & 6,486 \\
Water & $260 \cdot 5$ & 5,300 \\
Aqueous silicic acid* & $259 \cdot 0$ & $\mathbf{4 , 5 5 0}$
\end{tabular}

* When the solution stood for $24 \mathrm{~h}, \lambda_{\max }$ shifted to $257 \cdot 0\left(\varepsilon_{\max }=4,600\right)$ 